INPLASY

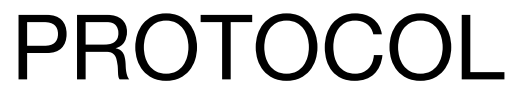

To cite: Li et al. Effect of multicomponent exercise on muscle strength, muscle endurance, and balance of frail older adults: A meta-analysis of randomized controlled trials. Inplasy protocol 2021110103. doi:

10.37766/inplasy2021.11.0103

Received: 29 November 2021

Published: 29 November 2021

Corresponding author:

Yao Li

ly140625@163.com

Author Affiliation:

The First Affiliated Hospital of China Medical University.

Support: China Medical University.

Review Stage at time of this submission: Data extraction.

Conflicts of interest:

None declared.

\section{Effect of multicomponent exercise on muscle strength, muscle endurance, and balance of frail older adults: A meta-analysis of randomized controlled trials}

Li, Y1; Hu, S2; Chen, H³ Zhang, M4; Liu, Y5.

Review question / Objective: What effect does multicomponent exercise have on the muscle strength, muscle endurance and balance of the frail older adults?

Condition being studied: Multicomponent exercise; Geriatrics; Nursing.

Information sources: We retrieved randomized controlled trials in English in the electronic databases including PubMed, CINAHL, Web of Science, Embase, Cochrane Library. The keywords according to the PICO framework.

INPLASY registration number: This protocol was registered with the International Platform of Registered Systematic Review and Meta-Analysis Protocols (INPLASY) on 29 November 2021 and was last updated on 29 November 2021 (registration number INPLASY2021110103).

\section{INTRODUCTION}

Review question / Objective: What effect does multi-component exercise have on the muscle strength, muscle endurance and balance of the frail older adults?

Condition being studied: Multicomponent exercise; Geriatrics; Nursing

\section{METHODS}

Search strategy: We retrieved randomized controlled trials in English in the electronic databases including Pubmed, CINAHL, Web of Science, Embase, Cochrane Library. Articles published from the inception of databases up to January 2021. The keywords according to the PICO 
framework including (Frail[MeSH] OR "Prefrail[Title/Abstract]") AND ("Older adults" OR "Older people" OR "Seniors" OR "Elderly" OR "Aged") AND ("Multicomponent* Exercise" OR "Multifactorial* Intervention" OR "Multimodal* Exercise" OR "Multisystem" Exercise" OR "Balance* Exercise" OR "Stability* Exercise" OR "Endurance* Exercise" OR "Aerobic* Exercise" OR "Stamina* Exercise" OR "Strength* Exercise" OR "Resistance* Exercise" OR "Physical Activity") AND ("Muscle Strength" OR "Muscle Endurance" OR "Muscle Mass" OR "Muscle Growth" OR "Muscle Power" OR "Balance"). Then further screening based on the abstract and the full text, the intervention measures must be multicomponent exercise with a combination of muscle strength, endurance, and balance, excluding single intervention measures (such as only balance exercise) research.

Participant or population: Frail and prefrail older adults.

Intervention: All studies including multicomponent exercise will be included in the intervention group

Comparator: Low-intensity exercise, a single exercise program such as resistance exercise only, daily activities

\section{Study designs to be included: RCTs}

Eligibility criteria: Inclusion criteria were the following: (a)Participants: older adults, aged 65 years or above, (b)Older adults living in community defined as prefrail and frail by the FRAIL scale and frailty phenotype; (c)Intervention measures: the intervention group applied multicomponent exercise intervention method that combined strength, balance and endurance exercise program (Sadjapong et al. 2020); the control group adopted routine lowintensity activities or remain their usual daily habits; (d)Type of study design: RCTs; (e)Language: published in English; (f)Outcome measures: In this study, we focused on the outcome indicators that multicomponent exercise has a significant impact on the physical function of frail elderly people by searching literature. Namely, muscle strength, muscle endurance and balance ability. Specifically, the outcome measures used in the primary studies were divided into 3 categories measured by objective indicators: (i)muscle strength (lower limb strength measured by knee extension strength (Kim et al. 2015) and upper limb strength measured by grip strength (Yu et al. 2020)); (ii)muscle endurance (measured by the chair-stand test(Rydwik et al. 2008) and VO2Max (Sadjapong et al. 2020)); (iii)balance (measured by one leg stand test (Rydwik et al. 2008)).Trials that contain the following criteria were excluded: (a)Incomplete data; (b)Duplicate publications; (c)Studies for which the outcome indicators were not reported or related outcome indicators could not be obtained even after contacting the authors; (d)Studies for which the abstract was available but the full text could not be retrieved.

Information sources: We retrieved randomized controlled trials in English in the electronic databases including PubMed, CINAHL, Web of Science, Embase, Cochrane Library. The keywords according to the PICO framework.

Main outcome(s): (i)muscle strength (lower limb strength measured by knee extension strength (Kim et al. 2015) and upper limb strength measured by grip strength (Yu et al. 2020)); (ii)muscle endurance (measured by the chair-stand test(Rydwik et al. 2008) and VO2Max (Sadjapong et al. 2020)); (iii)balance (measured by one leg stand test (Rydwik et al.2008)).

Quality assessment / Risk of bias analysis: Two researchers used Cochrane Handbook for Systematic Reviews of Interventions 5.1.0 (Higgins \& Green 2011) to evaluate the risk of bias, which mainly including 7 aspects of RCTs, that is allocation hiding, incomplete data reporting, blinding, selective reporting, and other biases. The evaluation is divided into three categories: low-risk bias, high-risk bias, and unclear. If there was any disagreement, negotiated with the third researcher to resolve. 
Strategy of data synthesis: This study will use RevMan 5.3 software to perform statistical analysis. We will estimate continuous data as weighted mean difference or standardized mean difference and $95 \%$ confidence intervals (CIs), dichotomous data as risk ratio and $95 \%$ Cls. We will identify statistical heterogeneity by $\mathrm{I}^{2}$ test. In accordance with the statistical heterogeneity levels among eligible studies, a fixed-effects model $\left(1^{2}\right.$ $\leq 50 \%)$ or a random-effects model $\left({ }^{2}>50 \%\right)$ will be applied to pool the extracted outcome data. If $I^{2} \leq 50 \%$, we will carry out a meta-analysis. Otherwise, if $\mathrm{I}^{2}>50 \%$, we will perform a subgroup analysis or sensitivity analysis to find possible sources from clinical and methodological aspects. We will conduct a narrative summary of study findings with supporting tables and figures, and will summarize narrative data reporting primary by describing the outcomes, methods of assessment, and provide preliminary assessment of the effective data.In this study, RevMan5.3 software was used to analyse the data. For outcomes measured with the same measurement tool, the mean difference(MD) and $95 \% \mathrm{Cl}$ were utilized, otherwise, the standardized mean difference(SMD) and $95 \% \mathrm{Cl}$ were calculated. Statistical heterogeneity between the studies was estimated by chisquare and 12 test. Given $P>0.1,12<50 \%$, it would be considered that there was no heterogeneity among the included trials, and fixed effect would be applied; on the contrary, if the included trials were considered to be heterogeneous, the random effect model would be applied, and analyzed the source of heterogeneity through sensitivity analysis or subgroup analysis.

Subgroup analysis: If necessary, we will carry out a subgroup analysis based on the study characteristics, details of treatment and controls, and outcomes.

Sensitivity analysis: If necessary, we will carry out a Sensitivity analysis.
Country(ies) involved: China.

Keywords: frail older adults; muscle strength; muscle endurance; balance.

Contributions of each author:

Author 1 - Yao Li.

Email: yli95@cmu.edu.cn

Author 2 - Shuang Hu.

Email: hys417501@163.com

Author 3 - Hongyu Chen.

Email: chyhkdyfy@163.com

Author 4 - Minyi Zhang.

Email: 772465105@qq.com

Author 5 - Yan Liu.

Email: cwx0609@sina.com

Language: English. 